\title{
Minimizing muscle fatigue through optimization of electrical stimulation parameters
}

\author{
Hossein Rouhani*1, Karen Elena Rodriguez ${ }^{2,3}$, Austin J Bergquist ${ }^{2,3}$, Kei Masani ${ }^{2,3}$, Milos R. Popovic ${ }^{2,3}$ \\ ${ }^{1}$ Department of Mechanical Engineering, University of Alberta, Edmonton, Alberta, Canada \\ ${ }^{2}$ Institute of Biomaterials and Biomedical Engineering, University of Toronto, Toronto, Ontario, Canada \\ ${ }^{3}$ Rehabilitation Engineering Laboratory, Lyndhurst Centre, Toronto Rehabilitation Institute - University Health Network, \\ Toronto, Ontario, Canada
}

Received: November 10, 2016

DOI: $10.5430 /$ jbei.v3n1p33
Accepted: December 13, 2016 Online Published: December 26, 2016

URL: http://dx.doi.org/10.5430/jbei.v3n1p33

\begin{abstract}
Rapid onset of muscle fatigue in response to electrical stimulation is a major challenge when designing a neuroprosthesis. This study aimed to introduce a decision-making algorithm to optimize pulse amplitude and pulse duration, for current regulated electrical stimulators, to attain a target joint torque level while minimizing muscle fatigue. We measured ankle torque produced by different pairs of pulse amplitude and pulse duration applied to the plantar-flexors. In each session, we measured the maximum generated torque and calculated muscle fatigue (fatigue time and torque-time integral). Then, we determined the pulse amplitude and pulse duration pair that generated a target level of torque while minimizing muscle fatigue. High bilateral symmetry and day-to-day repeatability was observed for the torque time-series between the left and right plantar-flexors of each participant (median correlation coefficient $=0.95$ ). Compared to the average fatigue obtained by various pulse amplitude and pulse duration pairs for a given level of torque, delivering pulses with the optimal pair reduced fatigue on average by $22.5 \%$ according to fatigue time and $6.6 \%$ according to torque-time integral. We created an empirical model describing how pulse amplitude and pulse duration can be modulated to generate specific levels of plantar-flexion torque with minimum muscle fatigue.
\end{abstract}

Key Words: Electrical stimulation, Pulse amplitude, Pulse duration, Muscle fatigue, Neuroprosthesis

\section{INTRODUCTION}

Functional electrical stimulation (FES) has been widely used to generate contractions and improve motor function in people who experience paralysis due to spinal cord injury or stroke. ${ }^{[1]}$ FES is used in two distinct manners; i) as a permanent orthotic device (i.e. neuroprosthesis) to permanently substitute lost motor function such as grasping, ${ }^{[1,2]}$ walking $^{[3,4]}$ or standing, ${ }^{[5,6]}$ and ii) as a short-term therapeutic intervention to help improve voluntary control of impaired function such as reaching, grasping ${ }^{[7]}$ and walking. ${ }^{[8]}$ How- ever, a major challenge in any FES application is rapid muscle fatigue, defined here as a reduction in torque with continuous activation.

Unlike the way motor units are recruited during voluntary contractions, FES does not recruit motor units according to the size principle whereby slow, fatigue-resistant motor units are recruited before fast, fatigable motor units. ${ }^{[9,10]}$ Rather, FES leads to random recruitment of both fast and slow motor units, according to the distance and orientation of terminal axon branches relative to the surface stimulating

\footnotetext{
*Correspondence: Hossein Rouhani; Email: hrouhani@ualberta.ca; Address: 10-368 Donadeo Innovation Centre for Engineering, $9211-116$ Street NW, Edmonton, AB T6G 1H9, Canada.
} 
electrodes. ${ }^{[1]}$ Thus, for a given contraction level, relatively more fatigable motor units contribute during FES compared with voluntary contractions. Also, unlike voluntary contractions, during which motor units fire asynchronously with respect to each other at relatively low rates $(<20 \mathrm{~Hz}$ for soleus $\left.^{[12]}\right)$, FES evokes synchronous recruitment of motor units and, therefore, requires higher stimulation frequencies $(>20 \mathrm{~Hz})$ to generate fused contractions. ${ }^{[11]}$ Lastly, during voluntary efforts, contraction levels can be maintained by either modulating the firing rates of active motor units or by recruiting separate motor units as the initially active motor units become fatigued.$^{[13,14]}$ Such complex temporal and spatial recruitment strategies are not possible with current FES technologies, whereby the same predominantly superficial motor units are continuously activated. ${ }^{[15,16]}$ Such altered motor unit recruitment is thought to underlie the enhanced metabolic demand and exaggerated muscle fatigue that occurs during FES, compared with voluntary efforts. ${ }^{[17]}$

When FES is applied, the torque generated at a joint depends on the characteristics of the individual electrical pulses, namely the pulse frequency, pulse amplitude (PA) and pulse duration (PD). It is known that fatigue in general increases with pulse frequency. ${ }^{[1,18]}$ Therefore, in practice, pulse frequency is usually fixed at a sufficiently low value to minimize fatigue, yet high enough to generate fused contractions. However, the effect of PA and PD on fatigue is inconclusive. ${ }^{[19,20]}$ In order to control FES-evoked contraction levels, typical commercially available stimulators allow researchers and rehabilitation practitioners to regulate PA (or PD) and to maintain PD (or PA) constant. ${ }^{[21,22]}$ Pulse charge $(\mathrm{PA} \times \mathrm{PD})$ is a main determinant of motor unit recruitment. Increasing the PA produces a stronger depolarising drive that travels deeper into the underlying tissue ${ }^{[23]}$ and recruits a greater number of motor units, ${ }^{[16]}$ whereas adjusting the PD influences not only the number of recruited motor units, but may also influence the selectivity of motor unit recruitment, due to threshold differences that exist between axons of different diameter lying at the same distance from the stimulating electrodes. ${ }^{[24-26]}$ Thus, it may be possible to apply different combinations of PA and PD that generate contractions of equivalent torque, yet differing fatigue-resistance, through the activation of somewhat separate populations of motor units based on differences in the location, and threshold, of axons relative to the FES electrodes. With this in mind, the purpose of the present experiments was to determine whether optimal PA and PD combinations (i.e., optimal (PA,PD) pairs), those that minimize fatigue, differ for separate levels of generated torque. The present study examined how the interplay of PA and PD can be optimized to minimize fatigue during FES. The final product of which is a protocol that can be used to calculate optimal (PA,PD) pairs for separate levels of generated torque during constant-frequency (rectangular pulse) FES.

\section{Methods}

\subsection{Participants}

Three human participants $(27.3 \pm 4.7$ years, $64 \pm 10 \mathrm{~kg}$, $164 \pm 8 \mathrm{~cm}, 1$ female) with no known neurological or musculoskeletal impairment volunteered for the present study. The local Ethics committee approved this study and participants gave their informed consent prior to taking part. Each participant attended nine experimental sessions with at least three days separating consecutive sessions.

\subsection{Experimental setup}

Participants sat comfortably on a padded bench with their feet positioned horizontally and secured to separate footplates (see Figure 1). Both foot-plates were attached by a shaft to a torque transducer (TS11-200 Flange Style Reaction Torque Transducer, Interface Inc, USA). The isometric plantar-flexion torque, applied to the foot-plates, was directly measured using this toque sensor on the shaft. Therefore, a mechanical model of the ankle lever arms and collection of the relevant anthropometric data were not required. Since the calculation of the ankle torque, rather than muscle force, would be needed in applications such as neuroprosthesis, measurement and analysis of muscle force was not required. The participant's thighs were tightly fixed on the bench, such that the shanks were kept vertical with respect to foot-plates.

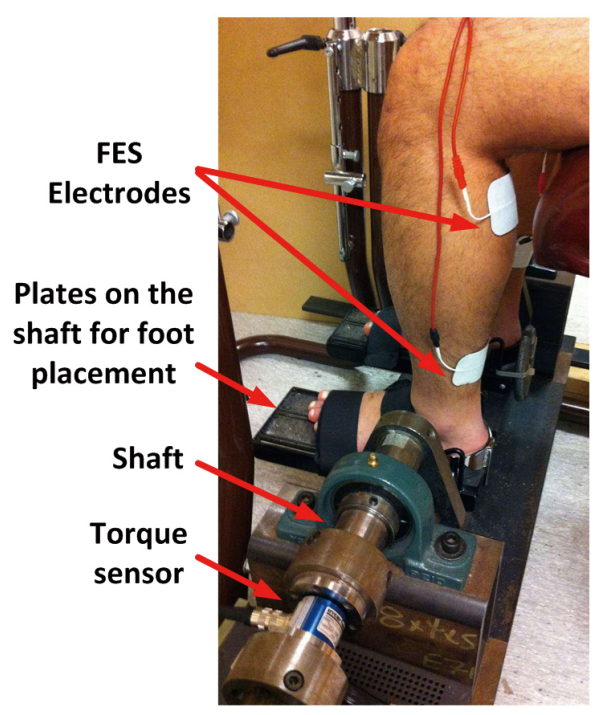

Figure 1. A system to measure isometric ankle joint torque generated by FES. Participant's feet were secured to foot-plates connected to a torque transducer via a shaft. 


\subsection{Functional electrical stimulation (FES)}

FES was applied through a pair of surface electrodes $(5 \times$ $9 \mathrm{~cm}$; STIMTRODE, model ST5090, Nidd Valley Medical Ltd, UK) placed along the midline of the ankle plantar-flexor muscles. One electrode was placed below the knee, $\sim 2 \mathrm{~cm}$ below the popliteal fossa, over the gastrocnemius and soleus motor points. The other electrode was placed proximal to the Achilles tendon over the distal end of the soleus muscle belly. Electrode locations were precisely determined and marked with respect to the anatomical landmarks and the electrodes were placed on the marked locations in the next sessions to ensure inter-session repeatability. An electrical stimulator (Compex Motion II, Compex SA, $\mathrm{CH}$ ) provided current regulated biphasic, asymmetric, balanced rectangular pulses (pulse delivering the charge had an amplitude of PA and a duration of PD, and the pulse removing the charge had an amplitude of PA/4 and a duration $4 \times \mathrm{PD}$ ). ${ }^{[27,28]}$

At the beginning of each session, PA and PD were adjusted, and then a constant train of rectangular pulses (frequency = $25 \mathrm{~Hz}$ ) was delivered separately to each leg for $180 \mathrm{sec}$ to generate ankle torque. According to our preliminary observations, the frequency of $25 \mathrm{~Hz}$ for stimulation pulse trains was low enough to avoid rapid onset of muscle fatigue and high enough to generate tetanic (fused) muscle contraction that is intended in most of FES applications. Other researchers have used frequencies in similar ranges. ${ }^{[29-31]}$ In each session, the PA was set to 30,40 , or $50 \mathrm{~mA}$, and the PD was set to 150,300 , or $450 \mu \mathrm{sec}$, producing 9 (PA,PD) pairs $(3 \times 3)$ that were tested in separate sessions. Based on our previous observation, these ranges of PA and PD generate measureable ankle torque that is well-tolerated, while larger PAs or longer PDs either generate torque that saturate or that is not well-tolerated by participants due to discomfort from the stimulation. The order of PA and PD levels was independently randomized for each participant and each leg. In each session, FES was delivered to the left leg first. Since the isometric torque was measured and the feet had minimal motion on the foot-plates, the effect of contra-lateral leg's inertia was minimal. In addition, the subjects were instructed to avoid voluntary contraction on the contra-lateral plantar-flexors According to technical limitations in our experimental setup, we were not able to record the reflex activation of contralateral extensors, and thus were not able to assess its effects on the recorded torque.

\subsection{Observational data acquisition and analysis}

Data were sampled at $100 \mathrm{~Hz}$ using a custom-made data acquisition system consisting of a data acquisition board (DAQ Multifunction NI USB-6211, National Instruments Corp., USA) and custom-written LabVIEW software (National Instruments Corp., USA). During FES, ankle plantar-flexion Published by Sciedu Press torque was recorded as a function of time. From this torque data, we measured the maximum torque (MT) generated over the entire stimulation time $(180 \mathrm{sec})$ and we calculated 2 indices of muscle fatigue: i) fatigue time (FT) representing the time difference from when MT was achieved to when torque dropped to $70.7 \%$ of MT (3 dB drop) and ii) torque-time integral (TTI) representing the integral of torque over the entire stimulation time, normalized to the maximum torquetime integral (MT $\times$ stimulation-time). This normalization was performed to avoid the influence of stimulation time on the calculated TTI.

The torque time-series produced by the left and right plantarflexors, for each participant, in response to the same (PA,PD) pair were compared using Pearson's correlation coefficient to assess bilateral symmetry and day-to-day repeatability of our experimental protocol. In addition, inter-participant repeatability of the torque time-series was assessed using coefficient of multiple correlations (CMC) for the left and right plantar-flexors obtained by the same (PA,PD) pair. ${ }^{[32]}$ Pearson's correlation coefficients were calculated to test the strength of the relationship between the injected electrical charge $(\mathrm{PA} \times \mathrm{PD})$ and 3 parameters: i) MT, ii) FT, and iii) TTI. Pearson's correlation coefficients were also calculated to test the strength of the relationship between MT and 2 parameters: i) FT and ii) TTI. Finally, Pearson's correlation coefficients were calculated to test the strength of the relationship between FT and TTI. Significance was set at $p<$ .05 .

\subsection{Mathematical modeling and analysis}

First the observed MT, FT and TTI values were combined on a grid based on the 3 levels of PA and 3 levels of PD tested presently. Then, additional MT, FT, and TTI values were calculated by interpolating over this grid using cubic spline, thereby generating continuous surfaces of (PA,PD,MT), (PA,PD,FT), and (PA,PD,TTI) in 3-dimensional space (see Figures 2a, 2b, and 2c, respectively). Any level of target MT $\left(\mathrm{MT}_{\text {target }}\right)$ can be generated using a variety of (PA,PD) pairs that form an iso-torque curve, (PA, $\mathrm{PD}, \mathrm{MT}_{\text {target }}$; see Figure 2a). This iso-torque curve is mathematically obtained by intersecting the $\mathrm{MT}=\mathrm{MT}_{\text {target }}$ plane with the (PA,PD,MT) surface. In Figure 2a, we have arbitrarily plotted the isotorque curve for a $\mathrm{MT}_{\text {target }}$ of $40 \mathrm{~N} . \mathrm{m}$. The corresponding curve can then be plotted on a fatigue index surface, FT (or TTI), to identify the FT (or TTI) values obtained for the selected (PA,PD) pairs (see Figure $2 b$ (or 2c)). The maximum FT (or TTI) value along this new curve was identified as the optimal (PA,PD) pair; see Figure $2 \mathrm{~b}$ for $(\mathrm{PA}, \mathrm{PD})_{O P T}$ on the FT surface and Figure 2c for (PA,PD) $O P T$ on the TTI surface. 

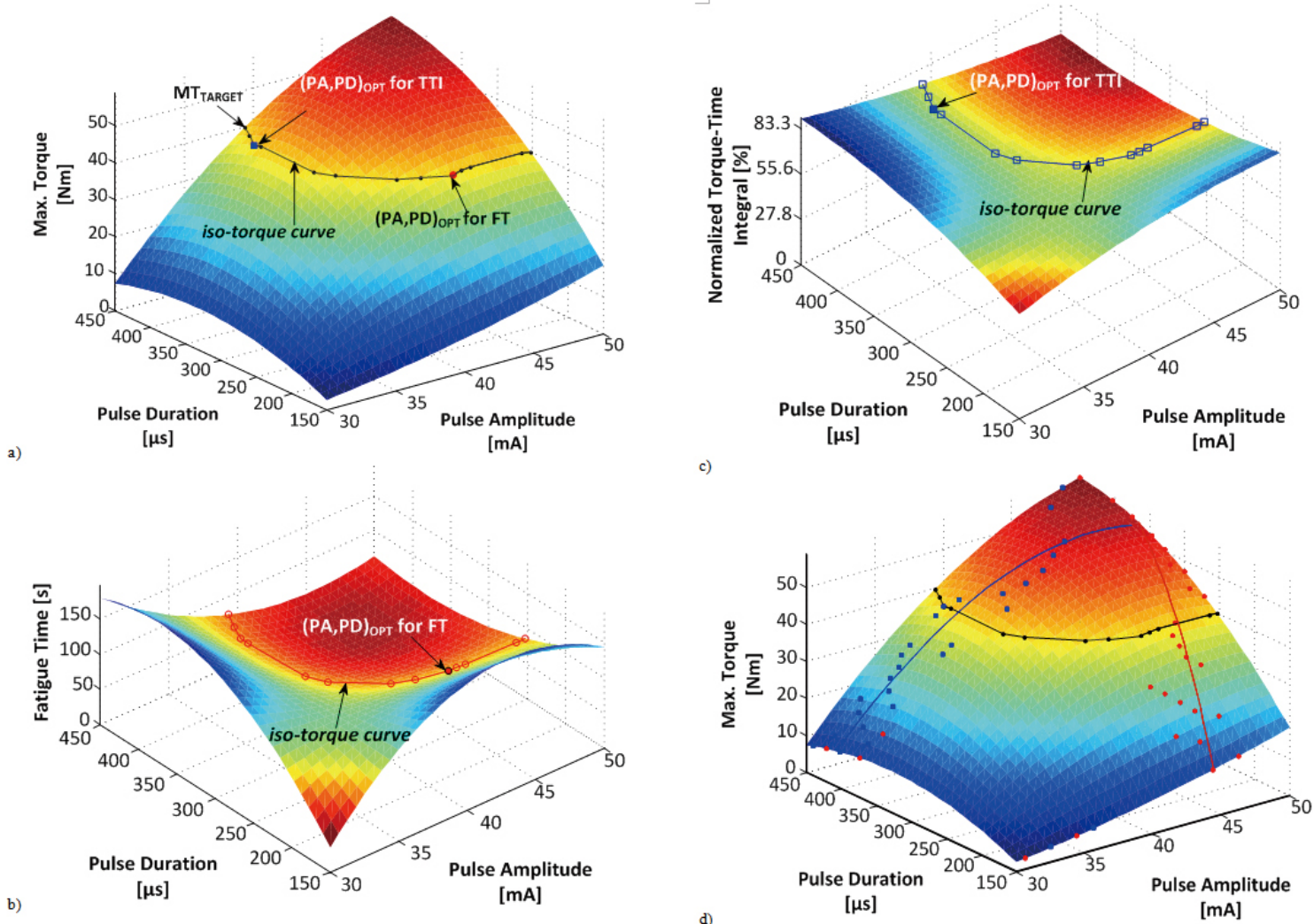

Figure 2. (a) Maximum torque (MT) as a function of pulse amplitude (PA) and pulse duration (PD). For any level of target maximum torque ( $\mathrm{MT}_{\text {target }}$ ) in FES applications (e.g., neuroprosthesis), an iso-torque curve is drawn on a MT surface indicating (PA,PD) pairs that generate $\mathrm{MT}_{\text {target }}$. See the following for the red circle and blue square. (b) The red curve represents fatigue time (FT) calculated for (PA,PD) pairs that are generating $\mathrm{MT}_{\text {target }}$ discussed in Figure 2a. Maximum FT on this red curve is shown by a red circle, indicating the (PA,PD) pair generating $\mathrm{MT}_{\text {target }}$ and resulting the longest fatigue time (i.e., least fatigue). The same red circle is also shown on the iso-torque curve in Figure (2a). (c) The blue curve represents the torque-time integral (TTI) normalized to the maximum torque-time integral (MT $\times$ stimulation time) calculated for $(\mathrm{PA}, \mathrm{PD})$ pairs that are generating $\mathrm{MT}_{\text {target }}$ discussed in Figure 2a. Maximum TTI on this blue curve is shown by a blue square, indicating the (PA,PD) pair generating $\mathrm{MT}_{\text {target }}$ and resulting in the largest TTI (i.e., least fatigue). The same blue square is also shown on the iso-torque curve in Figure 2a. (d) $\left(\mathrm{PA}^{i}, \mathrm{PD}^{i}\right)_{O P T}$ for FT (red) and $\left(\mathrm{PA}^{i}, \mathrm{PD}^{i}\right)_{O P T}$ for TTI (blue) points drawn on MT surface. These red and blue points indicate the (PA,PD) pairs that generate different levels of $\mathrm{MT}_{\text {target }}^{i}(i=1 . . \mathrm{N})$ and result in minimum muscle fatigue based on FT and TTI indices. The red and blue curves show the operating curves for optimal (PA,PD) pair modulation (projected on MT surface). These lines are calculated based on robust regression over the red circles and blue squares described in Figures $2 \mathrm{~b}$ and $2 \mathrm{c}$ for different torque levels.

Many implementations of FES (e.g., neuroprosthesis) require time-varying tunable torque. For this purpose, we calculated a sequence of $\left(\mathrm{PA}^{i}, \mathrm{PD}^{i}\right)_{O P T}(i=1 . . \mathrm{N})$ for $\mathrm{N}$ levels of $\mathrm{MT}_{\text {target }}^{i}(i=1 . . \mathrm{N})$, that minimize muscle fatigue based on FT (or TTI). An optimal way to tune MT in the target range is to simultaneously modulate $\mathrm{PA}$ and $\mathrm{PD}$ on an operating curve (line) passing through $\left(\mathrm{PA}^{i}, \mathrm{PD}^{i}\right)_{O P T}(i=1 . . \mathrm{N})$. In order to fit a curve through all $\left(\mathrm{PA}^{i}, \mathrm{PD}^{i}\right)_{O P T}(i=1 . . \mathrm{N})$, we produced a line according to robust regression; in Figure $2 \mathrm{~d}$, the red and blue lines represent the (PA,PD) pairs that minimize fatigue for a given level of $\mathrm{MT}_{\text {target }}$ according to FT and TTI, respectively.
To assess the efficiency of the fatigue optimization at each level of $\mathrm{MT}_{\text {target }}$ (i.e., along each iso-torque curve), we calculated the relative difference (\%) between the (PA,PD) pair that produced the maximum (optimal; least fatigue) and average (over all fatigue index values resulted from all (PA,PD) pairs corresponding to a given level of $\left.\mathrm{MT}_{\text {target }}\right)$ values. Then, we averaged the differences across all levels of $\mathrm{MT}_{\text {target }}$. This efficiency calculation was done separately for each fatigue index. Data analyses were performed using Matlab (Mathworks Inc, USA). 


\section{Results}

Figure 3 shows torque time-series in response to FES $(3 \times 3$ levels of (PA,PD)) applied to the left plantar-flexors of a single participant in 9 separate sessions. Usually, when large amounts of charge, based on the (PA,PD) levels, was injected, MTs were reached within the first $30 \mathrm{sec}$. The (PA,PD) pairs of ( $40 \mathrm{~mA}, 150 \mu \mathrm{sec}),(30 \mathrm{~mA}, 300 \mu \mathrm{sec})$, and $(30 \mathrm{~mA}$, $450 \mu \mathrm{sec}$ ) showed extremely long FT (virtually no fatigue). For these (PA,PD) pairs, the torque never dropped to $70.7 \%$ of its maximum value and, thus, we considered FT as the end of $180 \mathrm{sec}$ of stimulation (as the longest time we measured). Although FT is still shown (red square in Figure 3), and very close to MT, the recorded FT was $180 \mathrm{sec}$ (as shown in the plots of Figures 2 and 4 for these (PA,PD) pairs). The
(PA,PD) pair of $(30 \mathrm{~mA}, 150 \mu \mathrm{sec})$ generated small torque, and thus, the precision of the FT and TTI calculations were not high due to small signal-to-noise ratios. Figure 4 shows the interpolated (PA,PD,MT), (PA,PD,FT) and (PA,PD,TTI) surfaces for the left and right plantar-flexors of the same participant as in Figure 3. In general, as PA and PD increased, MT increased while FT and TTI values decreased, except for the $(30 \mathrm{~mA}, 150 \mu \mathrm{sec})$ pair. Figure 2 shows the interpolated (PA,PD,MT), (PA,PD,FT) and (PA,PD,TTI) surfaces for the left plantar-flexors of the same participant as in Figure 3 and 4. Importantly, (PA,PD) pairs that generated equal $\mathrm{MT}_{\text {target }}$ (i.e., pairs along the same iso-torque curve) differed in the level of fatigue produced over 3 min of FES (see Figures $2 b$ and 2c).
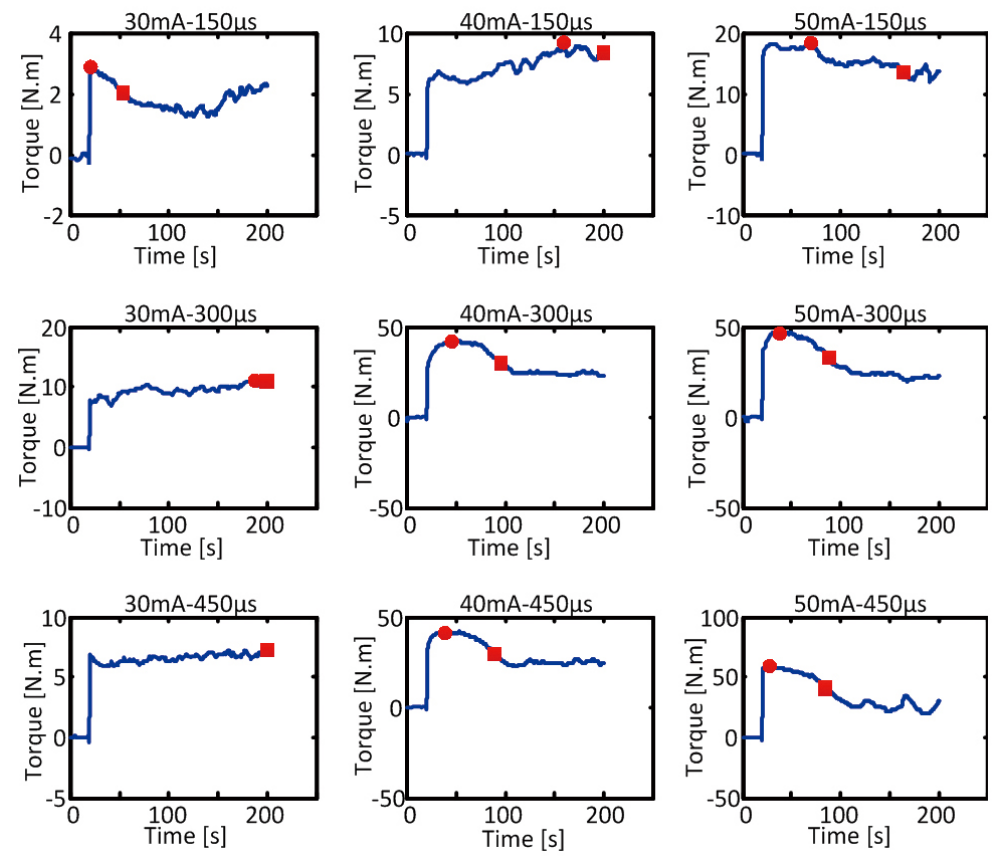

Figure 3. Nine torque time-series from a representative participant generated by $3 \times 3$ levels of PA and PD applied to the left plantar-flexors of the same participant as in Figure 2. The red circle shows the maximum torque (MT) generated and the red square shows the point where toque dropped to $70.7 \%$ of MT, indicating the fatigue time (FT).

Table 1 reports Pearson correlation coefficients $(\mathrm{R})$ for torque time-series obtained for each (PA,PD) pair between the left and right plantar-flexors of each participant obtained on different days (from 3 to 27 days apart). These values had a median of 0.95 over all 3 participants and all 9 (PA,PD) pairs, showing high bilateral symmetry and day-to-day repeatability. Table 2 reports the inter-participant repeatability of the measured torque time-series. Although the inter-session repeatability between the left and right plantar-flexors of each participant was high (see Table 1), the inter-participant repeatability was low (i.e., participants had very different torque time-series for a given (PA,PD) pair). The coefficients of multiple correlations (CMC) between the participants for torque time-series obtained for each (PA,PD) pair had a median of 0.71 averaged over all 9 (PA,PD) pairs and both plantar-flexors (see Table 2). CMC values were lower for higher levels of PD and PA (see Table 2). MT was significantly correlated with injected charge $(\mathrm{PA} \times \mathrm{PD})$, while FT and TTI were not significantly correlated with either PA $\times$ PD or MT (see Table 3). Compared the average fatigue resulted by different pairs of (PA,PD) for a given MT, delivering FES with the optimal $(\mathrm{PA}, \mathrm{PD})$ pair of a given $\mathrm{MT}\left((\mathrm{PA}, \mathrm{PD})_{O P T}\right)$ reduced fatigue by $22.5 \%$ according to FT and $6.6 \%$ according to TTI, on average across each participant, plantar-flexor 
and level of MT (see Table 4). The contribution of our methodology to fatigue minimization negatively correlated $(p<.03)$ with the level of MT (correlation coefficient averaged over all subjects: -0.53 and -0.61 , for FT and TTI, respectively). Note that correlation analysis for fatigue pa- Table 1).
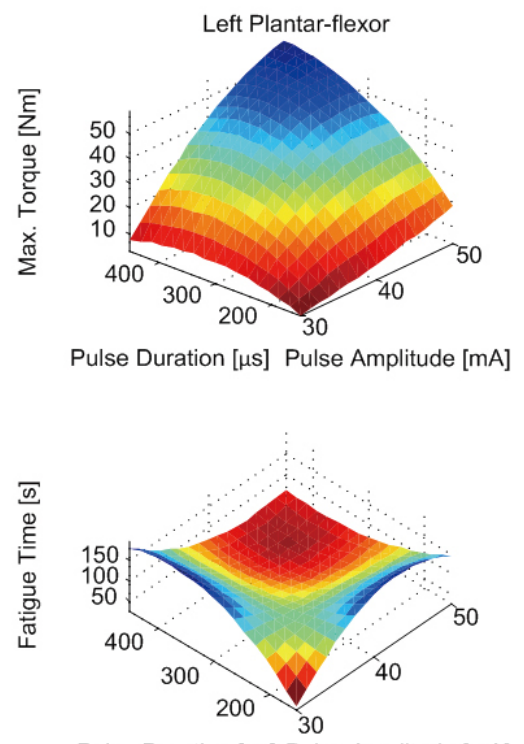

Pulse Duration [ $\mu \mathrm{s}]$ Pulse Amplitude [mA]

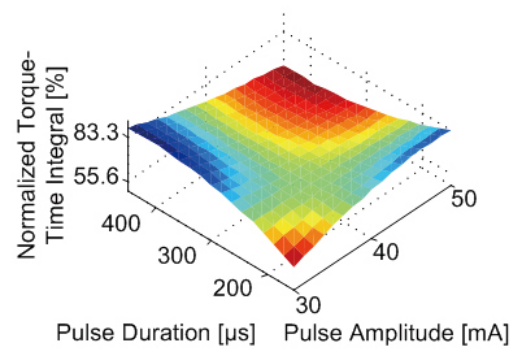

rameters of the left and right plantar-flexors in Tables 3 and 4 were performed separately. This was because the fatigue behavior of the left and right plantar-flexors of each subject for each stimulation condition were not independent (see

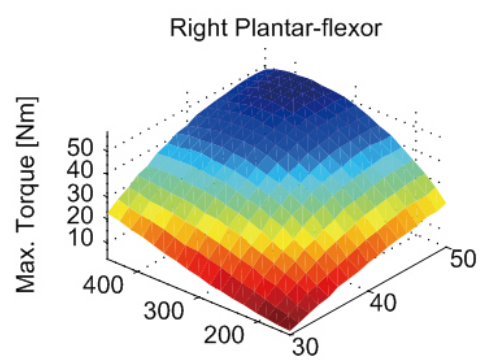

Pulse Duration [ $\mu \mathrm{s}]$ Pulse Amplitude [mA]

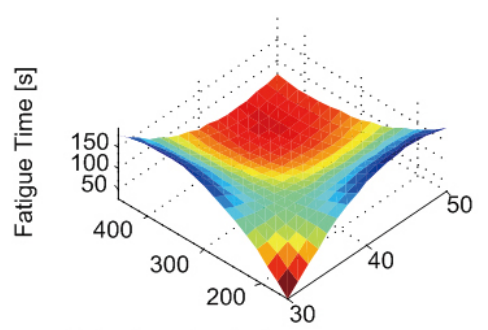

Pulse Duration [ $\mu \mathrm{s}]$ Pulse Amplitude [mA]

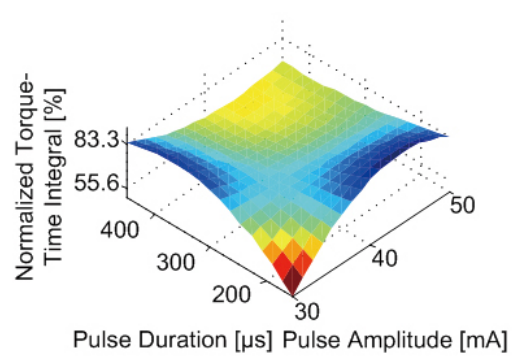

Figure 4. Maximum torque (MT), fatigue time (FT), and torque-time integral (TTI) surfaces based on interpolation of the 9 torque time-series generated by $3 \times 3$ levels of PA and PD applied to the left and right plantar-flexors the same participant as in Figures 2 and 3.

Table 1. Pearson correlation coefficients (R) for the measured torque time-series between the left and right plantar-flexors of each participant. All correlation coefficients were significantly different from zero at the 0.05 levels.

\begin{tabular}{lllll}
\hline $\begin{array}{l}\text { Pulse Amplitude } \\
\text { [mA] }\end{array}$ & $\begin{array}{l}\text { Pulse Duration } \\
\text { [ } \boldsymbol{\mu s}]\end{array}$ & \multicolumn{3}{l}{$\begin{array}{l}\text { Correlation Coefficients for } \\
\text { each participant }\end{array}$} \\
\cline { 3 - 5 } & & $\mathbf{S 1}$ & $\mathbf{S 2}$ & $\mathbf{S 3}$ \\
\hline \multirow{3}{*}{30} & 150 & 0.83 & 0.57 & 0.77 \\
& 300 & 0.96 & 0.89 & 0.81 \\
& 450 & 0.98 & 0.97 & 0.68 \\
40 & 150 & 0.96 & 0.92 & 0.38 \\
& 300 & 0.99 & 0.97 & 0.57 \\
& 450 & 0.98 & 0.97 & 0.96 \\
50 & 150 & 0.77 & 0.83 & 0.68 \\
& 300 & 0.98 & 0.90 & 0.98 \\
& 450 & 0.97 & 1.00 & 0.95 \\
\hline
\end{tabular}

Table 2. Coefficients of multiple correlations (CMC) for the measured torque time-series among 3 participants (to express the inter-participant repeatability). All coefficients of multiple correlations were significantly different from zero at the 0.05 levels.

\begin{tabular}{llll}
\hline $\begin{array}{l}\text { Pulse Amplitude } \\
{[\mathbf{m A}]}\end{array}$ & $\begin{array}{l}\text { Pulse Duration } \\
{[\boldsymbol{\mu s}]}\end{array}$ & \multicolumn{2}{c}{ CMC among participants } \\
\cline { 3 - 4 } 30 & 150 & Left ankle & Right ankle \\
\hline \multirow{3}{*}{30} & 300 & 0.81 & 0.77 \\
& 450 & 0.80 & 0.78 \\
& 150 & 0.75 & 0.76 \\
40 & 300 & 0.84 & 0.88 \\
& 450 & 0.33 & 0.69 \\
& 150 & 0.28 & 0.19 \\
50 & 300 & 0.26 & 0.80 \\
& 450 & 0.12 & 0.35 \\
& & 0.21 & 0.38 \\
\hline
\end{tabular}


Table 3. a) Pearson correlation coefficients (R) between the injected electrical charge $(\mathrm{PA} \times \mathrm{PD})$ and other measured parameters: i) maximum generated torque (MT), ii) fatigue time (FT) and iii) torque-time integral (TTI), averaged across $3 \times 3$ stimulation conditions and 3 participants. b) Pearson correlation coefficients between MT and other measured parameters: i) FT and ii) TTI, averaged across $3 \times 3$ stimulation conditions and 3 participants. c) Pearson correlation coefficients between fatigue indices: FT and TTI, averaged across $3 \times 3$ stimulation conditions and 3 participants. (*) Significance set at $p<0.05$.

\begin{tabular}{|l|l|l|}
\hline a) & Left ankle & Right ankle \\
\hline Measured parameter & $\mathbf{0 . 5 7 *}$ & $\mathbf{0 . 4 5}$ \\
\hline Maximum Torque (MT) & -0.06 & 0.21 \\
\hline Fatigue Time (FT) & -0.04 & 0.31 \\
\hline Torque-Time Integral (TTI) & Left ankle & Right ankle \\
\hline b) & 0.08 & 0.05 \\
\hline Measured parameter & 0.05 & 0.28 \\
\hline Fatigue Time (FT) & \multicolumn{2}{|l}{} \\
\hline Torque-Time Integral (TTI) & Left ankle & Right ankle \\
\hline c) & $\mathbf{0 . 5 2 *}$ & $\mathbf{0 . 8 3}$ \\
\hline Measured parameter & \\
\hline $\begin{array}{l}\text { Fatigue Time (FT) Torque-Time } \\
\text { Integral (TTI) }\end{array}$ &
\end{tabular}

Table 4. Efficiency of fatigue optimization, calculated as the relative difference $(\%)$ between the $(\mathrm{PA}, \mathrm{PD})$ pair that produced the maximum (Optimal; least fatigue) and average (over all values of a fatigue index resulted from all (PA,PD) pairs corresponding to a given level of $\mathrm{MT}_{\text {target }}$ ) values at each level of $\mathrm{MT}_{\text {target }}$, averaged across all levels of $\mathrm{MT}_{\text {target }}$. The efficiency calculation was repeated separately for both fatigue indices (Index: FT or TTI).

\begin{tabular}{llll}
\hline & & \multirow{2}{*}{$100 \times \frac{\text { Optimal(Index) }- \text { Average(Index) }}{\text { Average(Index) }}$} \\
\hline Participant & Side & FT & TTI \\
S1 & Left & 46.2 & 10.2 \\
& Right & 18.5 & 6.8 \\
S2 & Left & 14.7 & 4.5 \\
& Right & 26.4 & 7.5 \\
S3 & Left & 23.0 & 5.2 \\
& Right & 6.4 & 5.3 \\
\hline \multicolumn{2}{l}{ Mean (participant/side) } & $\mathbf{2 2 . 5}$ & $\mathbf{6 . 6}$ \\
\hline
\end{tabular}

\section{DISCUSSION}

This study investigated how to select pulse amplitude (PA) and pulse duration (PD) pairs that generate target plantarflexor torque levels while minimizing muscle fatigue during FES. Our approach involved: i) measuring the maximum generated torque (MT) and calculating common fatigue indices (fatigue time (FT) and torque-time integral (TTI)) in response to $3 \times 3$ levels of PA and PD; ii) interpolating MT, FT and TTI values from the observed data; iii) identifying (PA,PD) pairs that generated equivalent torque (i.e., iso-torque curves); iv) determining the optimal (PA,PD) pair that minimized muscle fatigue for each iso-torque curve according to FT or TTI; v) proposing a protocol of simultaneous (PA,PD) pair regulation to generate target torque levels while minimizing fatigue.

\subsection{Optimal selection of PA and PD}

\subsubsection{Simultaneous modulation of $P A$ and $P D$}

The red and blue lines in Figure 2d show the (PA,PD) modulation strategies that minimized fatigue based on FT and TTI indices, respectively, for a single participant. To produce a given level of joint torque ( $\left.\mathrm{MT}_{\text {target }}\right)$ with FES, there were distinct optimal (PA,PD) pairs depending on the functional goal: i) one pair generates torque that remains above the desired threshold the longest (located on the red line), and ii) another pair for which the torque integral over time will be the largest (located on the blue line). We expected continuity of the optimized red and blue points around the red and blue lines, respectively. The outliers may be due to multiple local maximums identified on iso-torque lines in lower levels of MT (see Figure 4), because of the inaccuracy of the fitted surface on the 9 experimental FT and TTI values. We expect that conducting the experiment with $4 \times 4$ data points (or more) instead of $3 \times 3$ would minimize these outliers. We applied "robust regression" instead of "ordinary regression" to increase the robustness of the results to such outliers. In the future, sensitivity of the obtained optimal operating lines to different sources of errors should be further investigated.

\subsubsection{Separate modulation of PD or PA}

The present results indicate that the optimal solution for generating a variety of target torques ( $\left.\mathrm{MT}_{\text {target }}\right)$, while minimizing fatigue, requires that both PA and PD be modulated simultaneously. However, many commercially available stimulators allow modulation of only PA or PD separately. For such stimulators that cannot modulate both PA and PD simultaneously, the present results also indicate that a nearly optimal performance can be achieved by simply setting PA (or PD) constant while modulating PD (of PA), depending on the desired torque profile. For example, considering the data for a particular subject in Figure 2d, we recommend distinct strategies: i) fix PA at a higher level, (from 43 to $50 \mathrm{~mA}$, in the vicinity of the red line), and modulate PD to generate torques that produce minimum fatigue according to FT, or ii) fix PD at a higher level, (from 340 to $440 \mu \mathrm{sec}$, in the vicinity of the blue line), and modulate PA to generate torques that produce minimum fatigue according to TTI. Note that these strategies are suggested for only a particular subject and different strategies maybe suggested for others. The physiological mechanism underlying the separate modulation strategies for optimizing FT and TTI are not at present clear. 
In our study, the minimum and maximum levels of PA and PD were chosen based on their threshold of measurable torque generation and maximum limits of tolerable discomfort, respectively. Our rationale was to cover the entire range of practical PA and PD utilized in the design of neuroprosthesis. Although the addition of an extra measurement point would increase the accuracy of our results, the absence of fatigue observed with the lowest levels of PA and PD would not necessarily affect the reliability of our experiment.

\subsection{Repeatability of results and clinical implementation}

\subsubsection{Inter-session repeatability}

Experiments with the left and right plantar-flexors of each participant with each (PA,PD) pair was performed 3 to 27 days apart. The lack of similarity (indicated by low correlation coefficient) between the generated torque time-series by the left and right plantar-flexors would indicate the lack of either bilateral symmetry or day-to-day repeatability. In our study, in contrast, high correlation (median $R=0.95$ ) was observed between the generated torque time-series produced by FES using different (PA,PD). This indicated both high bilateral symmetry and day-to-day repeatability of the experiments simultaneously. Therefore, the proposed strategy for regulating (PA,PD) pairing for each participant can be re-used later for the same participant. Consequently, our control strategy for each participant is sufficiently consistent and thus suitable for repetitive clinical applications. However, since muscle training can affect FES performance, ${ }^{[33]}$ it is expected that muscle training will change the proposed strategy for modulating (PA,PD) pairing, and thus the present experimental protocol should be repeated at regular intervals following prolonged FES training.

\subsubsection{Inter-participant repeatability}

The generated torque time-series produced by FES using different (PA,PD) pairs showed low inter-participant repeatability (median $\mathrm{CMC}=0.71$; see Table 2 ). As a result, the optimal strategy for modulating (PA,PD) pairing differed between participants, and thus we recommend that the optimal strategy for modulating (PA,PD) pairing be determined separately for each person participating in FES programming. The main source of inter-participant variability is due to differences in muscle fatigue among the participants, especially at higher levels of injected charge $(\mathrm{PA} \times \mathrm{PD}$; see Table 2).

\subsubsection{Correlations among electrical charge $(P A \times P D), M T$, FT and TTI}

It is known that torque generated by FES depends on the injected electrical charge $(\mathrm{PA} \times \mathrm{PD})$. However, it is unclear whether muscle fatigue is also a function of $\mathrm{PA} \times \mathrm{PD}$, rather than a more complicated function of (PA,PD) pairing. Presently, MT was significantly correlated with $\mathrm{PA} \times \mathrm{PD}$ (see
Table 3). However, neither FT nor TTI showed significant correlation with either PA $\times$ PD or MT. This observation indicates that, although MT varies proportionally with $\mathrm{PA} \times \mathrm{PD}$, all (PA,PD) pairs with identical PA $\times$ PD did not necessarily result in the same level of fatigue (assessed by FT or TTI). Thus, it is relevant to determine the (PA,PD) pairing that produces minimum fatigue. In addition, although FT and TTI indicate different fatigue resistance characteristics and resulted in different optimal operating lines, they were significantly correlated together. This indicates that FT and TTI both quantify similar general trends in muscle fatigue and are convergent indicators of fatigue resistance.

\subsubsection{Efficiency of fatigue optimization}

The present results confirm, as alluded to in the Introduction section, that different (PA,PD) pairs can be applied that generate contractions of equivalent MT, yet differing fatigueresistance. This finding is likely due to each (PA,PD) pair activating somewhat separate motor unit populations that have different inherent fatigue characteristics (i.e., recruiting relatively more or less slow, fatigue-resistant motor units). Importantly, the optimal (PA,PD) pairing differed depending on the level of generated torque (MT) and fatigue indices. Rehabilitation practitioners can expect an average improvement of $22.5 \%$ and $6.6 \%$ in fatigue-resistance according to FT and TTI, respectively. The contribution of our methodology to fatigue reduction negatively correlated with the level of MT. According to Figures 2 and 4, there are a fewer number of (PA,PD) pairs that can result in higher levels of torque, compared to lower torque levels. Further, there was less variability in fatigue indices among all (PA,PD) pairs at higher levels of MT. As such, there was less fatigue improvement through our suggested optimization for higher torque levels compared to lower torque levels.

\subsection{Limitations}

\subsubsection{Choice of muscle fatigue index}

Our proposed methodology can be applied to minimize fatigue based on any chosen fatigue index and does not recommend for or against any particular fatigues index. Presently, we applied our protocol to FT and TTI, as two previously used fatigues indices in the literature. ${ }^{[18,20,33-37]}$ A drop of MT from its maximum (100\%) to $70.7 \%$ (3 dB) was chosen since, in the control engineering literature, when the system output drops by $3 \mathrm{~dB}$, its performance is considered altered. ${ }^{[33]}$ This value has been used in other studies. ${ }^{[18,38]}$ Nevertheless, other researchers have also used a threshold of $50 \% .^{[34,39,40]}$

Because of different mathematical definition of FT and TTI, different obtained optimized operating lines are expected; mathematically various FT can be observed with a single 
value of TTI (or vice versa). Notably, while both FT and TTI significantly correlate together and assess fatigue resistance, they describe different aspects of fatigue resistance behavior. Different applications may demand use of either of these two fatigue indices, and subsequently either of the two optimized (PA,PD) pairs. FT indicates the capability of maintaining a threshold level of torque (70.7\% of its maximum value) within a certain time. FT is a relevant fatigue index for applications in which torque should not drop below a certain level, as such a drop could compromise system safety (e.g., FES-assisted walking). On the other hand, TTI indicates the capability of maintaining an average level of torque in the long-term. In applications where the average torque produced over a time period is important, it is more appropriate to use TTI as a fatigue index (e.g., FES-assisted grasping).

In the present results, at lower levels of injected $\mathrm{PA} \times \mathrm{PD}$, a low signal-to-noise ratio combined with low security in torque production resulted in low bilateral symmetry (see Table 1) and higher sensitivity to measurement error (e.g., when low torque levels $[<10 \mathrm{Nm}]$ are generated in Figure 3). However, our measurement precision was sufficiently high at higher levels of injected $\mathrm{PA} \times \mathrm{PD}$, where both torque production and fatigue were substantial that has higher clinical relevance.

\subsubsection{Sample size}

The sample size of the present study $(n=3)$ was relatively small. Indeed, we did not expect to have high interparticipant repeatability. Given the wide range of variability between individuals with paralyzed or paretic muscles in terms of age, body mass, gender, and the type and level of neuromuscular impairment, muscle atrophy, etc., we expect high inter-subject variability in their muscle response. Therefore, high inter-subject repeatability of muscle response to FES, and thus similar optimal operating lines (see Figure 4), is not expected. Our results confirm that even able-bodied subjects do not show similar muscle response to FES, mainly because of their different muscle training and the high variability in spatial motor unit recruitment between participants reported previously. ${ }^{[18]}$ Therefore, increasing the number of subjects would not result in obtaining a universal optimal (PA,PD) modulation strategy, expressed as red and blue lines in Figure 4, for all subjects. The primary goal of this study was to present a protocol that can be applied on an individual basis, and thus any larger sample size would not affect the final conclusions of this study.

\subsubsection{Population and generalizability}

The present study is a first step in understanding the complex relationship that exists between modulation of FES parameters (PA and PD), torque production and fatigue. Indeed, the present findings may not generalize to people with motor impairment. In fact, it is expected that the modulation of optimal (PA,PD) pairings will differ between and within populations other than that tested presently. Further, modulation of optimal (PA,PD) pairings likely differ for other stimulation paradigms (present findings were based on 180 sec of constant uninterrupted stimulation) and other muscles. However, the aim of this study was to present a protocol ( $\times \mathrm{n}$ PA and PD pairing) that allows for the calculation of optimal (PA,PD) pairings, which can itself be applied to other populations and other muscle groups.

Therefore, the feasibility of identifying optimal (PA,PD) pairings in people with motor impairment is excepted to be equally achievable. However, the importance of identifying optimal (PA,PD) pairings may be less pronounced, particularly in populations who experience chronic paralysis due to spinal cord injury, as motor unit populations have been shown to transition to a more homogenous fast-fatigable phenotype. ${ }^{[41]}$ As such, selective motor unit activation is expected to be less effective in this population. That said, previous work has shown that delivering stimulation to the chronically paralyzed plantar-flexors in ways known to recruit fatigue-resistant motor units (i.e., through reflex pathways) showed improvements in fatigue-resistance compared to conventional stimulation. ${ }^{[42]}$ This indicates that even in the chronic stage of recovery after spinal cord injury, there still exists a range of motor units that are resistant to fatigue, and as such, selective activation of motor units through identifying (PA,PD) pairings may also be important in this population.

Currently, it takes 27 days to determine an optimal operating line for each individual. This is not ideal for a clinical setting. Alternative approaches may reduce the total testing time to even a few days, for example by utilizing shorter stimulation time. In the future, alternative approaches to reduce the testing time could be developed for facilitating clinical application.

\section{Conclusion}

We found that different pairings of pulse duration (PD) and pulse amplitude (PA) can produce identical plantar-flexion torques, with differing levels of fatigue-resistance. Specifically, we presented a method to empirically calculate these optimal (PA,PD) pairs as a function of time to optimize either fatigue time (FT) or torque-time integral (TTI). We showed that optimal (PA,PD) pairings are stable if participants do not change his/her daily exercise regimen. We also found that these optimal (PA,PD) pairings are participant dependent and, thus, should be determined for each participant independently before neuroprostheses or other FES therapies 
are applied. Although we have demonstrated this method using the ankle plantar-flexor muscles, the same method may be used for other muscles or muscle groups to determine optimal stimulation parameters.

\section{ACKNOWLEDGeMents}

Hossein Rouhani was supported by the Swiss National Science Foundation Grant (PBELP3-137539 and P300P2-
147865/1) and the Spinal Cord Injury Ontario Foundation. This work was also partly supported by the Canadian Institute of Health Research (CIHR: Grants no. MOP-111225 and MOP-97952), the Natural Sciences and Engineering Research Council (NSERC, Canada: Grant no. 249669). The authors acknowledge the support of the Toronto Rehabilitation Institute - University Health Network.

\section{REFERENCES}

[1] Popovic MR, Curt A, Keller T, et al. Functional electrical stimulation for grasping and walking: Indications and limitations. Spinal Cord. 2001; 39(8): 403-412. PMid:11512070. https://doi.org/10.1 038/sj.sc. 3101191

[2] Malešević NM, Maneski LZP, Ilić V, et al. A multi-pad electrode based functional electrical stimulation system for restoration of grasp Journal of NeuroEngineering and Rehabilitation. 2012; 9(1): 66.

[3] Graupe D, Cerrel-Bazo H, Kern H, et al. Walking performance, medical outcomes and patient training in FES of innervated muscles for ambulation by thoracic-level complete paraplegics. Neurological Research. 2008; 30(2): 123-130. PMid:18397602. https: //doi.org/10.1179/174313208X281136

[4] Bulea TC, Kobetic R, Audu ML, et al. Stance controlled knee flexion improves stimulation driven walking after spinal cord injury. Journal of NeuroEngineering and Rehabilitation. 2013; 10: 68. PMid:23826711. https://doi .org/10.1186/1743-0003-1 0-68

[5] Fisher LE, Miller ME, Bailey SN, et al. Standing after spinal cord injury with four-contact nerve-cuff electrodes for quadriceps stimulation. IEEE Transactions on Neural Systems and Rehabilitation Engineering. 2008; 16(5): 473-478. PMid:18990650. https : //doi.org/10.1109/TNSRE. 2008.2003390

[6] Triolo RJ, Bailey SN, Miller ME, et al. Longitudinal performance of a surgically implanted neuroprosthesis for lower-extremity exercise, standing, and transfers after spinal cord injury. Archives of Physical Medicine and Rehabilitation. 2012; 93(5): 896-904. PMid:22541312. https://doi.org/10.1016/j.apmr.2012.01.001

[7] Kowalczewski J, Gritsenko V, Ashworth N, et al. Upper-Extremity Functional Electric Stimulation-Assisted Exercises on a Workstation in the Subacute Phase of Stroke Recovery. Archives of Physical Medicine and Rehabilitation. 2007; 88(7): 833-839. PMid:17601461. https://doi.org/10.1016/j.apmr.2007.03.036

[8] Thrasher TA, Flett HM, Popovic MR. Gait training regimen for incomplete spinal cord injury using functional electrical stimulation. Spinal Cord. 2006; 44(6): 357-361. PMid:16249784. https : //doi.org/10.1038/sj.sc.3101864

[9] Henneman E, Somjen G, Carpenter DO. Functional significance of cell size in spinal motoneurons. Journal of Neurophysiology. 1965; 28: 560-580. PMid:14328454.

[10] Milner-Brown HS, Stein RB, Yemm R. The orderly recruitment of human motor units during voluntary isometric contractions. Journal of Physiology. 1973; 230(2): 359-370. PMid:4350770. https : //doi.org/10.1113/jphysiol.1973.sp010192

[11] Gregory CM, Bickel CS. Recruitment patterns in human skeletal muscle during electrical stimulation. Physical Therapy. 2005; 85(4): 358-364. PMid:15794706.
[12] Bellemare F, Woods JJ, Johansson R, et al. Motor-unit discharge rates in maximal voluntary contractions of three human muscles. Journal of Neurophysiology. 1983; 50(6): 1380-1392. PMid:6663333.

[13] Carpentier A, Duchateau J, Hainaut K. Motor unit behaviour and contractile changes during fatigue in the human first dorsal interosseus. Journal of Physiology. 2001; 534(3): 903-912. https : //doi.org/10.1111/j.1469-7793.2001.00903.x

[14] Bawa P, Pang MY, Olesen KA, et al. Rotation of motoneurons during prolonged isometric contractions in humans. Journal of Neurophysiology. 2006; 96(3): 1135-1140. PMid:16775202. https : //doi.org/10.1152/jn.01063.2005

[15] Okuma Y, Bergquist AJ, Hong M, et al. Electrical stimulation site influences the spatial distribution of motor units recruited in tibialis anterior. Clinical Neurophysiology. 2013; 124(11): 2257-2263. PMid:23692977. https://doi.org/10.1016/j.clinph. 2013. 04.015

[16] Adams GR, Harris RT, Woodard D, et al. Mapping of electrical muscle stimulation using MRI. Journal of Applied Physiology. 1993; 74(2): 532-537. PMid:8458767.

[17] Theurel J, Lepers R, Pardon L, et al. Differences in cardiorespiratory and neuromuscular responses between voluntary and stimulated contractions of the quadriceps femoris muscle. Respiratory Physiology and Neurobiology. 2007; 157(2-3): 341-347. PMid:17210271. https://doi.org/10.1016/j.resp.2006.12.002

[18] Nguyen R, Masani K, Micera S, et al. Spatially distributed sequential stimulation reduces fatigue in paralyzed triceps surae muscles: A case study. Artificial Organs. 2011; 35(12): 1174-1180. PMid:21501192. https://doi .org/10.1111/j.1525-1594.2010.01195.x

[19] Gorgey AS, Black CD, Elder CP, et al. Effects of electrical stimulation parameters on fatigue in skeletal muscle. Journal of Orthopaedic and Sports Physical Therapy. 2009; 39(9): 684-692. PMid:19721215. https://doi.org/10.2519/jospt.2009.3045

[20] Thrasher A, Graham GM, Popovic MR. Reducing muscle fatigue due to functional electrical stimulation using random modulation of stimulation parameters. Artificial Organs. 2005; 29(6): 453-458. PMid:15926981. https ://doi .org/10.1111/j.1525-1594. 20 05.29076. $\mathrm{x}$

[21] Kobravi HR, Erfanian A. A decentralized adaptive fuzzy robust strategy for control of upright standing posture in paraplegia using functional electrical stimulation. Medical Engineering and Physics. 2012; 34(1): 28-37. PMid:21764350. https://doi.org/10.101 6/j.medengphy. 2011.06.013

[22] Same M, Rouhani H, Masani K, et al. Closed-loop control of ankle plantarflexors and dorsiflexors using an inverted pendulum apparatus: A pilot study. Journal of Automatic Control. 2013; 21: 31-36. https://doi.org/10.2298/JAC1301031S

[23] Mesin L, Merlo E, Merletti R, et al. Investigation of motor unit recruitment during stimulated contractions of tibialis anterior muscle. 
Journal of Electromyography and Kinesiology. 2010; 20(4): 580-589. PMid:20044273. https ://doi.org/10.1016/j.jelekin. 2009 .11 .008

[24] Grill Jr WM, Mortimer JT. The effect of stimulus pulse duration on selectivity of neural stimulation. IEEE Transactions on Biomedical Engineering. 1996; 43(2): 161-166. PMid:8682527. https: //doi.org/10.1109/10.481985

[25] Gorman PH, Mortimer JT. The effect of stimulus parameters on the recruitment characteristics of direct nerve stimulation. IEEE Transactions on Biomedical Engineering. 1983; 30(7): 407-414. PMid:6604691.

[26] Szlavik RB, de Bruin H. The effect of stimulus current pulse width on nerve fiber size recruitment patterns. Medical Engineering \& Physics. 1999; 21(6-7): 507-515.

[27] Popovic MR, Keller T. Modular transcutaneous functional electrical stimulation system. Medical Engineering and Physics. 2005; 27(1): 81-92. PMid:15604009. https://doi.org/10.1016/j.medeng phy. 2004.08 .016

[28] Rouhani H, Popovic MR, Same M, et al. Identification of ankle plantar-flexors dynamics in response to electrical stimulation. Medical Engineering \& Physics. 2016; 38 (11): 1166-1171. PMid:27544922. https ://doi .org/10.1016/j .medengphy . 20 16.07 .011

[29] Kuhn A, Keller T, Lawrence M, et al. The Influence of Electrode Size on Selectivity and Comfort in Transcutaneous Electrical Stimulation of the Forearm. IEEE Trans. Neural Systems \& Rehabilitation Engineering. 2010; 18: 255-62.

[30] Kesar T, Binder-macleod S. Effect of frequency and pulse duration on human muscle fatigue during repetitive electrical stimulation. Experimetal Physiology. 2006; 91: 967-76. PMid:16873456. https://doi.org/10.1113/expphysiol.2006.033886

[31] Shields RK, Law LF, Reiling B, et al. Effects of electrically induced fatigue on the twitch and tetanus of paralyzed soleus muscle in humans. Journal of Applied Physiology. 1997; 82: 1499-507.

[32] Kadaba MP, Ramakrishnan HK, Wootten ME, et al. Repeatability of kinematic, kinetic, and electromyographic data in normal adult gait. Journal of Orthopaedic Research. 1989; 7(6): 849-860. PMid:2795325. https ://doi.org/10.1002/jor. 1100070611
[33] Graham GM, Thrasher TA, Popovic MR. The effect of random modulation of functional electrical stimulation parameters on muscle fatigue. IEEE Transactions on Neural Systems and Rehabilitation Engineering. 2006; 14(1): 38-45. PMid:16562630. https: //doi.org/10.1109/TNSRE. 2006.870490

[34] Thomas CK, Griffin L, Godfrey S, et al. Fatigue of paralyzed and control thenar muscles induced by variable or constant frequency stimulation. Journal of Neurophysiology. 2003; 89(4): 2055-2064 PMid:12611940. https://doi.org/10.1152/jn.01002.2002

[35] Shields RK, Dudley-Javoroski S. Musculoskeletal plasticity after acute spinal cord injury: effects of long-term neuromuscular electrical stimulation training. Journal of Neurophysiology. 2006; 95 : 2380-2390. PMid:16407424. https://doi.org/10.1152/jn.0 1181.2005

[36] Sayenko DG, Nguyen R, Hirabayashi T, et al. Method to reduce muscle fatigue during transcutaneous neuromuscular electrical stimulation in major knee and ankle muscle groups. Neurorehabilitation and Neural Repair 2015; 29: 722-733. PMid:25549655. https : //doi.org/10.1177/1545968314565463

[37] Enoka RM, Stuart DG. Neurobiology of muscle fatigue. Journal of Applied Physiology. 1992; 72: 1631-1648. PMid:1601767.

[38] Maneski L, Malesševic N, Savic A, et al. Surface-distributed lowfrequency asynchronous stimulation delays fatigue of stimulated muscles. Muscle Nerve. 2013; 448: 930-7. PMid:23512421. https : //doi.org/10.1002/mus. 23840

[39] Godfrey S, Butler JE, Griffin L. Differential Fatigue of Paralyzed Thenar Muscles by Stimuli of Different Intensities. Muscle Nerve. 2002; 26: 122-31. PMid:12115957. https://doi.org/10.1002/ mus. 10173

[40] Zonnevijlle ED, Somia NN, Stremel RW, et al. Sequential Segmental Neuromuscular Stimulation: An Effective Approach to Enhance Fatigue Resistance. Plast Reconstr Surg. 2000; 105: 667-73. https : //doi.org/10.1097/00006534-200002000-00028

[41] Grimby G, Broberg C, Krotkiewska I, et al. Muscle fiber composition in patients with traumatic cord lesion. Scandinavian Journal of Rehabilitation Medicine. 1976; 8: 37-42. PMid:132700.

[42] Bergquist AJ, Wiest MJ, Okuma Y, et al. H-reflexes reduce fatigue of evoked contractions after spinal cord injury. Muscle Nerve. 2014; 50 : 224-34. PMid:24638882. https ://doi.org/10.1002/mus . 2414 4 